\title{
A Late COVID-19 Complication: Male Sexual Dysfunction
}

\author{
Saeed Shoar, MD; ${ }^{1}$ (1) Siamak Khavandi, MD ${ }^{2}$ (1) Elsa Tabibzadeh, MD; ${ }^{3}$ Aydin Vaez, MD; ${ }^{4}$ \\ Ali Khabbazi Oskouei, MD $;^{5}$ Fatemeh Hosseini, MD; ${ }^{6}$ Mohammad Naderan, MD ${ }^{7}$ Nasrin Shoar, MD $^{8}$
}

1. Department of Clinical Research, ScientificWriting Corporation, Houston, Texas USA

2. Department of Ophthalmology, Tabriz University of Medical Sciences, Tabriz, Iran

3. Department of Anaesthesiology and Critical Care, Tabriz University of Medical Sciences, Tabriz, Iran

4. Department of Infectious Diseases, Tabriz University of Medical Sciences, Tabriz, Iran

5. Faculty of Medicine, Tabriz University of Medical Sciences, Tabriz, Iran

6. Faculty of Medicine, Isfahan University of Medical Sciences, Isfahan, Iran

7. Faculty of Medicine, Tehran University of Medical Sciences, Tehran, Iran

8. Faculty of Medicine, Kashan University of Medical Sciences, Tehran, Iran

\author{
Correspondence: \\ Saeed Shoar, MD \\ Department of Clinical Research \\ ScientificWriting Corporation \\ Houston, Texas USA \\ E-mail: saeedshoar@scientificwriting.org
}

Conflicts of interest: none

Keywords: anorgasmia; COVID-19; COVID-19 complication; SARS-CoV-2; sexual dysfunction

\section{Abbreviations:}

COVID-19: coronavirus infectious disease 2019

EC: emergency center

ICU: intensive care unit

PCR: polymerase chain reaction

SARS-CoV-2: severe acute respiratory syndrome novel coronavirus 2

Received: September 1, 2020

Revised: September 5, 2020

Accepted: September 13, 2020

\begin{abstract}
Since the beginning of the coronavirus infectious disease 2019 (COVID-19) pandemic, an exponentially large amount of data has been published to describe the pathology, clinical presentations, and outcomes in patients infected with the severe acute respiratory syndrome novel coronavirus 2 (SARS-CoV-2). Although COVID-19 has been shown to cause a systemic inflammation predisposing the involvement of multiple organs, its mechanism affecting the urogenital system has not been well-documented. This case report presents the clinical course of two male patients with COVID-19 who developed sexual dysfunction, as anorgasmia, following recovery from the infection. Although no evidence of viral replication or inflammatory involvement could be identified in these cases' urogenital organs, a lack of other known risk factors for anorgasmia points to the role of COVID-19 as the contributing factor.
\end{abstract}

Shoar S, Khavandi S, Tabibzadeh E, Vaez A, Oskouei AK, Hosseini F, Naderan M, Shoar N. A late COVID-19 complication: male sexual dysfunction. Prehosp Disaster Med. 2020;35(6):688-689.

Introduction

It has been close to six months since the World Health Organization (WHO; Geneva, Switzerland) announced the global spread of the severe acute respiratory syndrome novel coronavirus 2 (SARS-CoV-2) as a pandemic. ${ }^{1}$ An exponentially increasing number of studies were published describing the clinical presentations of coronavirus diseases 2019 (COVID-19). However, as the population continues to struggle with the emotional and physical burden of the pandemic, the need to increase attention to the quality of life of the patients who survive COVID-19 and enter a recovery period is apparent. This is of paramount importance considering the long duration of the pandemic and its exhausting impact on the health care system, as well as the well-being of the global population. Here, this case report presents an uncommon clinical symptom encountered by the two patients who uneventfully recovered from COVID- 19 .

\section{Case Presentation}

Case 1

A 44-year-old man with no previous medical history presented to the emergency center (EC) with a complaint of fever, chills, and frequent cough starting two weeks earlier. He had been in a home quarantine, but due to worsening symptoms and developing a shortness of breath, he decided to seek medical care. On admission, he had a transcutaneous oxygen saturation of $88 \%$ without supplementary oxygen, an oral temperature of $37.5^{\circ} \mathrm{C}$, and a respiratory rate of 18 breaths per minute. Lung auscultation showed reduced pulmonary sounds

\section{doi:10.1017/S1049023X20001223}

(C) The Author(s), 2020. Published by Cambridge University Press on behalf of World Association for Disaster and Emergency Medicine. This is an Open Access article, distributed under the terms of the Creative Commons Attribution-NonCommercial-NoDerivatives licence (http://creativecommons.org/ licenses/by-nc-nd/4.0/), which permits non-commercial re-use, distribution, and reproduction in any medium, provided the original work is unaltered and is properly cited. The written permission of Cambridge University Press must be obtained for commercial re-use or in order to create a derivative work. 
at the bases. A computerized tomography scan of the chest revealed bilateral ground-glass opacity of the lungs, consistent with COVID-19. Shortly after admission, he was intubated and underwent mechanical ventilation in the intensive care unit (ICU). During the first week of admission, he received three packs of cytosorb hemoperfusion dialysis along with hydroxychloroquine $200 \mathrm{mg}$ tablet daily and azithromycin $500 \mathrm{mg}$ tablet daily for seven days, five doses of subcutaneous $\beta$ interferon injection, high dose intravenous injection of corticosteroids (methylprednisolone $100 \mathrm{mg}$ daily), and melatonin $6 \mathrm{mg}$ tablet nightly. He also received vitamin supplementation (vitamin C 250mg tablet every six hours and vitamin B12 $1000 \mu \mathrm{g}$ daily). Following the improvement of symptoms, he was weaned off the ventilator seven days later and finally extubated. The next seven days in the medical floor were also uneventful and he was finally discharged home after 14 days of hospital stay. However, at the next follow-up survey via the phone, he complained of sexual dysfunction in the form of anorgasmia. Although there was no complaint of sexual dysfunction by the patient or his partner before the recent hospitalization, he seemed to develop this as a result of COVID-19 encounter. Interestingly, there was no decrease in the libido or a complaint of erectile dysfunction and the quality of sleep and nutritional status was normal. The patient did not mention stressful conditions and denied taking specific medications, using recreational drugs, or suffering psychiatric conditions. His wife had contracted COVID-19 one month earlier and recovered uneventfully. He was instructed to seek psychosexual consultation but he declined. A follow-up visit two weeks later at the clinic revealed complete resolution of patient condition.

\section{Case 2}

A 31-year-old man with a history of mild exertional asthma taking salbutamol spray occasionally presented to the $\mathrm{EC}$ with a complaint of fever, sore throat, cough, and shortness of breath starting five days prior. He had been in home quarantine for the past few days until his symptoms got worse. Vital signs were within normal limits, but a pulse oximetry showed a saturation of $85 \%$ without and $92 \%$ with the nasal oxygen. The patient was admitted to the ICU for further treatment. A CT of the chest, on the same day, showed bilateral lung base turbidity suggesting COVID-19. His treatment plan included azithromycin $500 \mathrm{mg}$ tablet once daily for five days, hydroxychloroquine tablet $200 \mathrm{mg}$ once daily for five days, and subcutaneous injection of $\beta$ interferon for five days. Respiratory therapy was provided during the hospital stay. Following maintenance of the oxygen saturation to above $92 \%$ without auxiliary oxygen, he was discharged home with no prescribed medications. During a follow-up visit three weeks later, patient complained of anorgasmia which had started since his discharge from the hospital.
However, he denied having a decreased libido or erectile dysfunction. His social history was unremarkable for illicit drug use or alcohol consumption. He had not been taking any medication over the last three months, except his course of hospitalization. Patient was referred to the sexual disorder and behavioral treatment clinic. However, he reported resolution of his symptoms 10 days later without any intervention. Notably, his sexual partner did not have a similar complaint although she had recovered from COVID-19 one month before her husband was admitted to the hospital.

\section{Discussion}

Extrapulmonary manifestations of COVID-19 have been increasingly reported in the literature. ${ }^{2,3}$ However, few studies have reported the pathophysiological involvement of SARS-CoV-2 in the male reproductive system. ${ }^{4,5} \mathrm{~A}$ study by La Marca, et al pointed out the potential involvement of testes in COVID-19 by reporting the testicular pain as the manifestation of COVID-19 in a 43-yearold man. ${ }^{5}$ However, the imaging and microbiological studies did not provide a proof of virus presence in the semen or testis. Similarly, previous studies utilizing polymerase chain reaction (PCR) techniques could not isolate SARS-CoV-2 in the genital secretions of individuals with laboratory-confirmed COVID-19., In contrast, these cases did not have any signs of genital involvement by SARS-CoV-2 and the only symptom was anorgasmia.

A cross-sectional study showed that COVID-19 pandemic, not the infection itself, has negatively impacted the sexual behavior of couples younger than the age of 35 years old. ${ }^{8}$ Of these, many individuals had a decreased sexual desire leading to lower frequency of sexual intercourse. However, sexual dysfunctions such as erectile dysfunction, anorgasmia, or premature ejaculation were not the scope of this investigation.

To the best of the authors' knowledge, this is the first report of anorgasmia in survivors of COVID-19. However, the long-term effects of infection with SRARS-Cov-2 on the sexual dysfunction is unknown. In addition, the underlying mechanism for such a sexual dysfunction is not known in patients with recent hospitalization due to COVID-19 but with a negative follow-up PCR, no post-hospital fatigue syndrome, or any impaired libido or psychosexual disturbances. Although a complex interaction of these stressful comorbidities might explain the occurrence of anorgasmia in survivors of COVID-19, future studies are warranted to investigate the known risk factors for sexual dysfunction in this patient population. As the COVID-19 pandemic is extending to the flu season, increasing attention toward the quality of life of patients surviving this infection is of paramount importance, no need to underscore the sexual well-being as a vital contributor.
References

1. Centers for Disease Control and Prevention. New ICD-10-CM code for the 2019 Novel Coronavirus (COVID-19). https:/www.cdc.gov/nchs/data/icd/AnnouncementNew-ICD-code-for-coronavirus-3-18-2020.pdf?fbclid=IwAR1W4E21-xZbEJdSGRFewVZmuM72GGhiE2QIRyur_CPStp14uAa8gzhRXw\#: :text=On\%20March\% 2011\%2C\%202020\%20the,concerning\%20the\%20COVID\%2D19\%20Outbreak. Accessed August 14, 2020.

2. Behzad S, Aghaghazvini L, Radmard AR, Gholamrezanezhad A. Extrapulmonary manifestations of COVID-19: radiologic and clinical overview. Clin Imaging. 2020; 66:35-41.

3. Wiersinga WJ, Rhodes A, Cheng AC, Peacock SJ, Prescott HC. Pathophysiology, transmission, diagnosis, and treatment of coronavirus disease 2019 (COVID-19): a review. JAMA. 2020;324(8):782-793.
4. Cardona Maya WD, Du Plessis SS, Velilla PA. SARS-CoV-2 and the testis: similarity with other viruses and routes of infection. Reprod Biomed Online. 2020;40(6):763-764.

5. La Marca A, Busani S, Donno V, Guaraldi G, Ligabue G, Girardis M. Testicular pain as an unusual presentation of COVID-19: a brief review of SARS-CoV-2 and the testis. Reprod Biomed Online. 2020. Epub ahead of print.

6. Paoli D, Pallotti F, Colangelo S, et al. Study of SARS-CoV-2 in semen and urine samples of a volunteer with positive naso-pharyngeal swab. J Endocrinol Invest. 2020. Epub ahead of print.

7. Xu J, Qi L, Chi X, et al. Orchitis: a complication of severe acute respiratory syndrome (SARS). Biol Reprod. 2006;74(2):410-416.

8. Li G, Tang D, Song B, et al. Impact of the COVID-19 pandemic on partner relationships and sexual and reproductive health: cross-sectional, online survey study. $J \mathrm{Med}$ Internet Res. 2020;22(8):e20961. 